\title{
Drug sensitivity in cancer cell lines is not tissue-specific
}

\author{
Samira Jaeger ${ }^{1 \dagger}$, Miquel Duran-Frigola ${ }^{1 \dagger}$ and Patrick Aloy ${ }^{1,2^{*}}$
}

\begin{abstract}
Background: Cancer cell lines have a prominent role in the initial stages of drug discovery, facilitating high-throughput screening of potential drugs. However, their clinical relevance remains controversial.

Findings: We assess whether drug sensitivity in cancer cell lines is able to discriminate tissue specificity. We find that cancer-specific drugs do not show higher efficacies in cell lines representing the respective tissues. Even when considering distinct cancer subtypes and targeted therapies, most drugs are evenly effective/ineffective throughout all cell lines.

Conclusions: To get the most out of cell line panels, it will be necessary to look into their molecular characteristics, and integrate them into systems biology frameworks.
\end{abstract}

Keywords: Cancer cell lines, Tissue specificity, Drug sensitivity

\section{Findings}

Human cancer cell lines are widely used in vitro models for studying cancer and its biology [1]. Apart from being valuable tools for identifying biomarkers and genetic variants that impact drug sensitivities [2,3], cancer cell lines play a pivotal role in the early stages of drug discovery, facilitating the screening of hundreds of potential drugs and their combinations before translating the outcomes into in vivo models and expensive clinical trials $[4,5]$.

However, despite their fundamental role in biomedical research, the clinical relevance of cell lines remains highly controversial [6,7]. Apart from known technical and biological limitations, like contamination, missing tumor microenvironment, or lack of drug distribution and metabolism, the main concern is whether cancer cell lines are true representatives of primary tumors [8]. Prolonged culturing of immortalized cell lines may induce extensive modifications in their molecular characteristics, like secondary genomic changes $[7,9]$, and it remains unclear how closely they still resemble the original tissue after undergoing a certain number of passages. To complement the

\footnotetext{
* Correspondence: patrick.aloy@irbbarcelona.org

${ }^{\dagger}$ Equal contributors

${ }^{1}$ Joint IRB-BSC-CRG Program in Computational Biology, Institute for Research in Biomedicine (IRB Barcelona), c/Baldiri i Reixac 10-12, Barcelona 08028, Catalonia, Spain

${ }^{2}$ Institució Catalana de Recerca i Estudis Avançats (ICREA), Pg. Lluís Companys 23, Barcelona 08010, Catalonia, Spain
}

ongoing debate from a pharmacological perspective, we analyzed the predictive power of cancer cell lines for elucidating cancer-specific drug responses. Considering the widely used NCI-60 panel [5], we assessed whether drugs for a particular cancer type display a higher efficacy in cell lines supposedly representing the respective cancer tissue. We focused on three cancer types, namely, breast, colorectal and prostate cancer, represented by five, seven and two cell lines, respectively, in the NCI-60. We analyzed all the 75 compounds, approved or experimental, associated with the treatment of these cancers that have also been tested in the NCI-60 (Additional file 1: Table S1 and Additional file 2: Table S2). We examined the sensitivity of each drug by considering its GI50 across the complete NCI-60 panel, derived from nine cancer tissues. The GI50, similar to IC50 and EC50, indicates the concentration required to inhibit cell proliferation by $50 \%$, relative to untreated samples [5]. Note that we studied the activity of final drugs instead of the preliminary hit compounds expecting that the former remain active after preclinical optimization. Figure 1A shows the sensitivity of the corresponding cancer cell lines towards cancer-specific agents (highlighted in red) in comparison to the sensitivity measured in cell lines representing other cancer tissues. Surprisingly, cancer-specific drugs do not show a significantly higher activity in cell lines representing the respective tissue (Wilcoxon test 


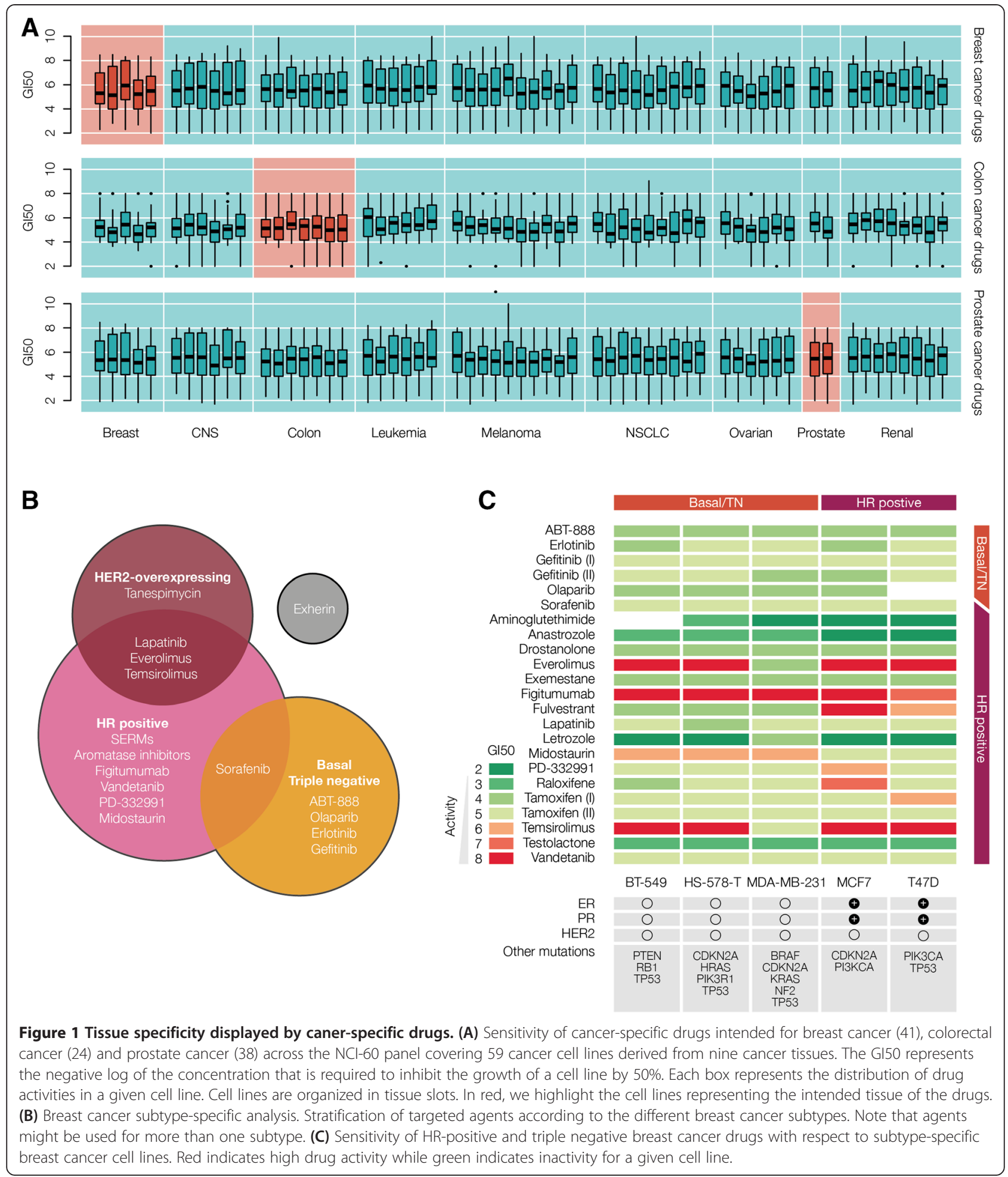

with Bonferroni correction for multiple testing; see Additional file 1). The same trend can be observed when focusing only on approved drugs or more specific targeted agents, and after normalizing drug sensitivity values across cell lines. To avoid biases caused by drugs with multiple therapeutic indications, we also analyzed more restrictive sets containing compounds exclusively associated with breast, colorectal or prostate cancers, obtaining equivalent outcomes (Additional file 1: Figures S1, S2 and S3). Overall, our results indicate that a simple 
selection of cell lines according to the tissue of interest does not reflect the eventual complexity in the clinics.

Given the intrinsic heterogeneity of most cancers [10], no individual cell line is likely to be a general representative for the distinct cancers derived from one tissue [11]. Hence, we do not expect a tissue-specific drug to perform equally well across the corresponding cell lines. Breast cancer, for instance, is a heterogeneous disease with at least four recognized subtypes that require a specific treatment $[12,13]$. Most researchers select particular cell lines based on receptor status, common genetic mutations, molecular signatures, tumor type, as well as technical or methodological limitations for their experiments [14]. Thus, mimicking this common approach, we considered the presence or absence of biomarkers that define the distinct breast cancer subtypes. Next we examined whether subtype-specific responses are reflected in cell lines representing distinct subtypes. The NCI-60 covers two subtypes, the triple-negative breast cancer (BT-549, HS-578 T and MDA-MB-231) and the HR-positive breast cancer subtype (MCF7 and T-47D). Neither the HER2overexpressing nor the normal-like subtypes are included in the panel. Among the 25 targeted breast-cancer agents, 18 are indicated for HR-positive patients, 6 for triplenegative and 4 for HER2-overexpressing breast cancer (Figure 1B and Additional file 1: Table S3). According to this stratification, we assessed the sensitivity of the subtype-specific drugs in the corresponding cell lines. Figure $1 \mathrm{C}$ shows that the majority of drugs is equally active or inactive independent of the breast cancer subtype. Figitumumab, vandetanib, and gefitinib (I), for example, are evenly effective in HR-positive and triplenegative cell lines, while aromatase inhibitors like letrozole, anastrozole and aminoglutethimide are basically inactive and would not have been discovered through screening cell lines from this panel. Only four out of 18 HR-positive drugs, i.e., PD-332991, raloxifene, tamoxifen (I), and fulvestrant, exhibit a higher specificity in at least one of the HR-positive cell lines. Conversely, midostaurin, a targeted drug against HR-positive tumors, only shows activity in triple-negative cell lines. As the NCI-60 only involves five breast cancer cell lines, which only partially reflect the distinct subtypes, we performed the same analysis on the more comprehensive Cancer Cell Line Encyclopedia (CCLE) [15]. Considering 30 breast cancer cell lines and 14 targeted agents, both stratified into subtypes, we still observe a largely varying compound sensitivity rather than subtype-specific responses in the corresponding cell lines (Additional file 1: Figure S4). Thus, even when accounting for subtype-specific differences in the NCI-60, or considering a much larger cell line panel with a broader representation of breast cancer subtypes [15] (see Additional file 1: Figure S4), the previously observed tendencies regarding tissue specificity still hold.
Similarly, we investigated whether intended drug targets are expressed in the corresponding cell lines and, if so, whether the drug is active in this cell line, exploiting proteomics data of NCI-60 [16]. Again, we could not correlate target expression with drug sensitivity (Additional file 1: Figure S5). One of the few exceptions is presented by the set of EGFR modulators, Gefitinib and Erlotinib, to which cell lines are indeed more sensitive when EGFR is expressed. Yet, in the majority of instances drug activity does not depend on the presence or absence of the intended targets, which indicates that several factors, beyond target expression, determine drug sensitivity.

Although alternative models for drug screening and development are generated [9], cancer cell lines have been and will be an essential component of cancer research and drug discovery [17]. However, as we observed, relying on assays performed in a few selected cell lines may result in incorrect or misleading conclusion, and thus is unlikely to predict clinical outcomes. Cell line panels, on the other hand, may embrace the underlying complexity and variability of cancer. Yet, to fully exploit their invaluable potential, we have to move beyond 'one marker, one cell line' studies and incorporate the large amount of molecular ('omics') profiles into robust systems biology frameworks $[15,16,18-20]$. We believe that identifying and combining the key features that each cell line is able to reproduce, beyond tissue and subtype specificity, will bring screening panels at the forefront of a more successful drug discovery.

\section{Additional files}

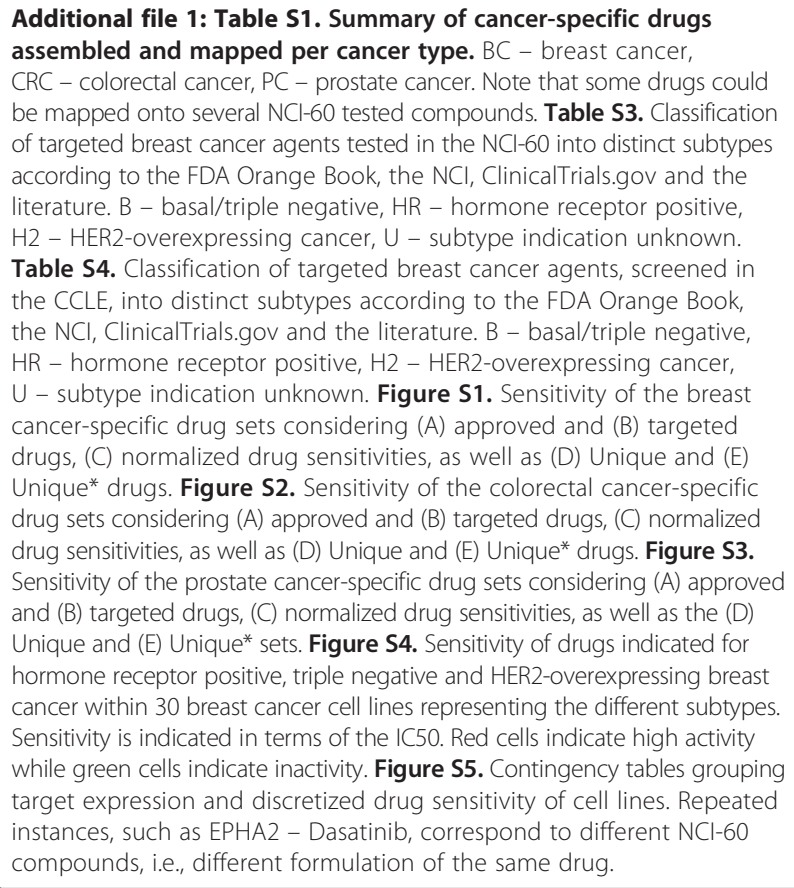


Additional file 2: Table S2. Overview on the cancer drugs assembled from DrugBank, TTD and the NCI for breast, colorectal and prostate cancer, including the mapping to $\mathrm{NCl}-60$ molecules and information on the drug status (approved), the agent type (targeted agents) and whether they are used to treat other diseases (Unique and Unique*).

\section{Competing interests}

The authors declare that they have no competing interest.

\section{Authors' contributions}

SJ, MDF and PA designed the research strategy. SJ and MDF performed the experiments. All the authors analyzed and discussed the results. SJ, MDF and PA wrote the manuscript. All authors read and approved the final manuscript.

\section{Acknowledgements}

We would like to thank all the IRB Barcelona PIs, specially E. Giralt and A.R. Nebreda, R. Mosca and the Biostatistics Unit for helpful discussions. This work was partially supported by the European commission through the SyStemAge project (Agreement n: 306240) and the European Research Council through the SysPharmAD grant (Agreement $n^{\circ}:$ 201014). MDF is a recipient of the Spanish FPU Fellowship.

Received: 11 November 2014 Accepted: 29 January 2015

\section{Published online: 15 February 2015}

\section{References}

1. Weinstein JN. Drug discovery: cell lines battle cancer. Nature. 2012;483:544-5.

2. Garnett MJ, Edelman EJ, Heidorn SJ, Greenman CD, Dastur A, Lau KW, et al Systematic identification of genomic markers of drug sensitivity in cancer cells. Nature. 2012:483:570-5.

3. Prahallad A, Sun C, Huang S, Di Nicolantonio F, Salazar R, Zecchin D, et al. Unresponsiveness of colon cancer to BRAF(V600E) inhibition through feedback activation of EGFR. Nature. 2012;483:100-3.

4. Gazdar AF, Girard L, Lockwood WW, Lam WL, Minna JD. Lung cancer cell lines as tools for biomedical discovery and research. J Natl Cancer Inst. 2010;102:1310-21.

5. Shoemaker $\mathrm{RH}$. The NCl60 human tumour cell line anticancer drug screen. Nat Rev Cancer. 2006;6:813-23.

6. Gazdar AF, Gao B, Minna JD. Lung cancer cell lines: useless artifacts or invaluable tools for medical science? Lung Cancer. 2010;68:309-18.

7. Gillet JP, Varma S, Gottesman MM. The clinical relevance of cancer cell lines. J Natl Cancer Inst. 2013;105:452-8.

8. Domcke S, Sinha R, Levine DA, Sander C, Schultz N. Evaluating cell lines as tumour models by comparison of genomic profiles. Nat Commun. 2013:4:2126.

9. Wilding $J$, Bodmer WF. Cancer cell lines for drug discovery and development. Cancer Res. 2014;74:2377-84.

10. Marusyk A, Polyak K. Tumor heterogeneity: causes and consequences. Biochim Biophys Acta. 1805;2010:105-17.

11. Vargo-Gogola T, Rosen JM. Modelling breast cancer: one size does not fit all. Nat Rev Cancer. 2007;7:659-72.

12. Perou CM, Sorlie T, Eisen MB, van de Rijn M, Jeffrey SS, Rees CA, et al. Molecular portraits of human breast tumours. Nature. 2000;406:747-52.

13. van 't Veer $L$, Dai H, van de Vijver MJ, He YD, Hart AA, Mao M, et al. Gene expression profiling predicts clinical outcome of breast cancer. Nature. 2002:415:530-6.

14. Holliday DL, Speirs V. Choosing the right cell line for breast cancer research. Breast Cancer Res. 2011;13:215

15. Barretina J, Caponigro G, Stransky N, Venkatesan K, Margolin AA, Kim S, et al. The cancer cell line encyclopedia enables predictive modelling of anticancer drug sensitivity. Nature. 2012:483:603-7.

16. Moghaddas Gholami A, Hahne H, Wu Z, Auer FJ, Meng C, Wilhelm M, et al. Global proteome analysis of the NCl-60 cell line panel. Cell Rep. 2013;4:609-20.

17. Ferreira D, Adega F, Chaves R. The Importance of Cancer Cell lines as in vitro Models in Cancer Methylome Analysis and Anticancer Drugs Testing. In: Lopez-Camarillo C, Arechaga-Ocampo E, editors. Oncogenomics and Cancer Proteomics - Novel Approaches in Biomarkers Discovery and Therapeutic Targets in Cancer. InTech. 2013.
18. Abaan OD, Polley EC, Davis SR, Zhu YJ, Bilke S, Walker RL, et al. The exomes of the NCl-60 panel: a genomic resource for cancer biology and systems pharmacology. Cancer Res. 2013;73:4372-82.

19. Ross DT, Scherf U, Eisen MB, Perou CM, Rees C, Spellman P, et al. Systematic variation in gene expression patterns in human cancer cell lines. Nat Genet. 2000;24:227-35.

20. Dancik GM, Ru Y, Owens CR, Theodorescu D. A framework to select clinically relevant cancer cell lines for investigation by establishing their molecular similarity with primary human cancers. Cancer Res. 2011;71:7398-409.

\section{Submit your next manuscript to BioMed Central and take full advantage of:}

- Convenient online submission

- Thorough peer review

- No space constraints or color figure charges

- Immediate publication on acceptance

- Inclusion in PubMed, CAS, Scopus and Google Scholar

- Research which is freely available for redistribution

Submit your manuscript at www.biomedcentral.com/submit
C Biomed Central 